REVISTA DE LA

UNIÓN MATEMÁTICA ARGENTINA

Vol. 59, No. 2, 2018, Pages 255-264

Published online: February 8, 2018

\title{
REMARKS ON LIOUVILLE-TYPE THEOREMS ON COMPLETE NONCOMPACT FINSLER MANIFOLDS
}

\author{
SONGTING YIN AND PAN ZHANG
}

Abstract. We give a gradient estimate of the positive solution to the equation

$$
\Delta u=-\lambda^{2} u, \quad \lambda \geq 0
$$

on a complete noncompact Finsler manifold. Then we obtain the corresponding Liouville-type theorem and Harnack inequality for the solution. Moreover, on a complete noncompact Finsler manifold we also prove a Liouville-type theorem for a $C^{2}$-nonnegative function $f$ satisfying

$$
\Delta f \geq c f^{d}, \quad c>0, d>1,
$$

which improves a result obtained by Yin and He.

\section{INTRODUCTION}

A Finsler space $(M, F, d \mu)$ is a differential manifold equipped with a Finsler metric $F$ and a volume form $d \mu$. The class of Finsler spaces is one of the most important metric measure spaces. Up to now, Finsler geometry has been developed rapidly in its global and analytic aspects. In [7], [10], [11], [14, [16] and [18, the study was well implemented on Laplacian comparison theorem, Bishop-Gromov volume comparison theorem and Liouville-type theorem, and so on.

In [13, Yau derived a gradient estimate for harmonic functions on complete, noncompact Riemannian manifolds with the Ricci curvature bounded below by negative constant and proved that complete Riemannian manifolds with nonnegative Ricci curvature must have Liouville property. Recently, the result was extended by Xia ([12]) to the Finsler manifolds under the condition that the weighted Ricci curvature has a lower bound, and by Zhang-Zhu ([17]) to the Alexandrov spaces.

Consider

$$
\Delta u=-\lambda^{2} u, \quad \lambda \geq 0
$$

on the Finsler manifold $(M, F, d \mu)$. Using the gradient estimate obtained in [12], we can give a gradient estimate of the positive solution to (1.1). This is inspired

2010 Mathematics Subject Classification. Primary 53C60; Secondary 53C24.

Key words and phrases. Liouville-type theorems; Finsler manifold; weighted Ricci curvature.

This paper is partially supported by AHNSF (No. 1608085MA03), NNSFC (No. 11471246) and TLXYRC (2015tlxyrc09). 
by the work by Ma ([3]) on similar result in Riemannian geometry. Specifically, we prove

Theorem 1.1. Let $(M, F, d \mu)$ be a complete noncompact Finsler n-manifold, equipped with a uniformly smooth and uniformly convex Finsler structure $F$. Assume that $\operatorname{Ric}_{N} \geq-K$ for some real numbers $N \in[n,+\infty)$ and $K \geq 0$. Let $u$ be a positive solution to 1.1 in a forward geodesic ball $B_{2 R}^{+}(p) \subset M$. Then there exists some constant $C=C\left(N, \Lambda_{1}, \Lambda_{2}\right)$, depending on $N$, the uniform constants $\Lambda_{1}$ and $\Lambda_{2}$, such that, in $B_{R}^{+}(p)$

$$
\max \{F(x, \nabla \log u(x)), F(x, \nabla(-\log u(x)))\} \leq C\left(\frac{1+R \sqrt{K}}{R}\right) .
$$

Using (1.2) we obtain the corresponding Liouville-type theorem and a Harnack inequality for the solution in Section 3 below. We remark that if $\lambda=0$ in (1.1), Theorem 1.1 becomes the main result in [12].

On the other hand, Nishikawa (4]) proved that if a $C^{2}$-nonnegative function $f$ satisfies $\Delta f \geq 2 f^{2}$ on a complete Riemannian manifold with Ricci curvature bounded from below, then $f$ vanishes identically. The result was extended by Choi, Kwon and Suh ([2]) to the general case $\Delta f \geq c f^{d}$ for $c>0, d>1$. Recently, Zhang ([15]) generalized it to Finsler manifolds if the weighted Ricci curvature $\operatorname{Ric}_{N} \geq-\widetilde{c}(\widetilde{c}>0)$. Jointly with He, the first author further generalized it under the condition that the weighted Ricci curvature is bounded from below by some function, and $F$ is reversible (see [14, Corollary 4.7]). Now we show that the last condition is redundant.

Theorem 1.2. Let $(M, F, d \mu)$ be a complete noncompact Finsler n-manifold, and $r(x)=d_{F}(p, x)$ be the distance function from a fixed point $p \in M$. Assume that the weighted Ricci curvature satisfies $\operatorname{Ric}_{N}(x, y) \geq-G^{2}(r(x)), \forall y \in T_{x} M, N \in[n, \infty)$, where $G$ is a smooth function satisfying

$$
G \geq 1, \quad G^{\prime} \geq 0, \quad \int_{0}^{\infty} \frac{d s}{G(s)}=\infty .
$$

If a nonnegative function $f \in C^{2}(M)$ satisfies

$$
\Delta f \geq c f^{d}, \quad c>0, d>1
$$

then $f$ vanishes identically.

Some definitions — such as those of Finsler manifold, the weighted Ricci curvature, gradient and Finsler Laplacian - will be given in Section 2 below. We remark that the Finsler gradient and Laplacian are nonlinear operators, which are much different from those on Riemannian manifolds. Besides, the results obtained do not coincide with those on weighted Riemannian manifolds, since the two kinds of weighted Ricci curvature $\operatorname{Ric}_{N}(x, y)$ and $\operatorname{Ric}_{N}^{\nabla u}$ are not the same.

To prove the theorems, we borrow some methods from the related literature (see [2], [3], [9]) and give them some adjustments. Precisely, let $\widetilde{M}=M \times \mathbb{R}$ be a Finsler $(n+1)$-manifold equipped with the product metric $\widetilde{F}=\sqrt{F^{2}+t^{2}}$; then its 
weighted Ricci curvature is also not less than $-K$. By setting $f(x, t)=e^{\lambda t} u(x)$ we find a harmonic function $f(x, t)$ on $\widetilde{M}$, and the gradient estimate is obtained from 12. Then 1.2 follows, as required. As to the proof of Theorem 1.2 we make full use of the relationship between the gradient and the reverse gradient, as well as the Finsler-Laplacian and the reverse Finsler-Laplacian of a function. Then the arguments can be followed step by step as in [2] (see also [14]).

\section{Preliminaries}

To meet the requirements in the next section, here some fundamentals of Finsler geometry are briefly presented.

Let $M$ be an $n$-dimensional smooth manifold and $\pi: T M \rightarrow M$ be the natural projection from the tangent bundle $T M$. A Finsler metric on $M$ is a function $F: T M \rightarrow[0,+\infty)$ satisfying the following properties:

(i) Regularity: $F$ is smooth in $T M \backslash 0$.

(ii) Positive homogeneity: $F(x, \lambda y)=\lambda F(x, y)$ for all $(x, y) \in T M$ and all $\lambda>0$.

(iii) Strong convexity: for every $(x, y) \in T M \backslash 0$, the matrix

$$
g_{i j}(x, y):=\frac{\partial^{2}}{\partial y^{i} \partial y^{j}}\left(\frac{1}{2} F^{2}\right)(x, y)
$$

is positive definite.

Such a pair $(M, F)$ is called a Finsler manifold. We say that $F$ is uniformly smooth and uniformly convex if there exist two uniform constants $0<\Lambda_{1} \leq \Lambda_{2}<\infty$ such that for $x \in M, y \in T_{x} M \backslash\{0\}$ and $W \in T_{x} M$,

$$
\Lambda_{1} F^{2}(x, W) \leq \sum_{i, j=1}^{n} g_{y}(W, W) \leq \Lambda_{2} F^{2}(x, W) .
$$

It is proved that a large class of Finsler manifolds satisfy the above property (see [6]).

Define the distance function on $(M, F)$ by

$$
d_{F}(p, q):=\inf _{\gamma} \int_{0}^{1} F(\gamma, \dot{\gamma}) d t
$$

where the infimum is taken over all differentiable curves $\gamma:[0,1] \rightarrow M$ with $\gamma(0)=p$ and $\gamma(1)=q$.

The reversibility $\eta$ of $(M, F)$ is defined by [8]

$$
\eta=\max _{X \in T M \backslash 0} \frac{F(X)}{F(-X)} .
$$

$(M, F)$ is called reversible if $\eta=1$. It is clear that the distance function $d_{F}$ of $F$ satisfies

$$
d_{F}(p, q) \leq \eta d_{F}(q, p), \quad \forall p, q \in M
$$


For every nonvanishing vector $V$ on an open set $U \subset M, g_{i j}(x, V)$ induces a Riemannian structure $g_{V}$ on $U$ via

$$
g_{V}(X, Y)=\sum_{i, j=1}^{n} g_{i j}(x, V) X^{i} Y^{j}, \quad \forall X, Y \in T_{x} U .
$$

In particular, $g_{V}(V, V)=F^{2}(x, V)$.

There exists a unique linear connection, which is called the Chern connection, on Finsler manifolds. The Chern connection is determined by the following structure equations, which characterize torsion freeness:

$$
D_{X}^{V} Y-D_{Y}^{V} X=[X, Y]
$$

and almost $g$-compatibility

$$
Z\left(g_{V}(X, Y)\right)=g_{V}\left(D_{Z}^{V} X, Y\right)+g_{V}\left(X, D_{Z}^{V} Y\right)+2 C_{V}\left(D_{Z}^{V} V, X, Y\right)
$$

for $V \in T U \backslash 0, X, Y, Z \in T U$. Here $C_{V}$ is the Cartan tensor given by

$$
C_{V}(X, Y, Z):=C_{i j k}(V) X^{i} Y^{j} Z^{k}=\frac{1}{4} \frac{\partial^{3} F^{2}}{\partial V^{i} \partial V^{j} \partial V^{k}}(\cdot, V) X^{i} Y^{j} Z^{k} .
$$

Given two linearly independent vectors $V, W \in T_{x} M \backslash 0$, the flag curvature is defined by

$$
K(V, W):=\frac{g_{V}\left(R^{V}(V, W) W, V\right)}{g_{V}(V, V) g_{V}(W, W)-g_{V}(V, W)^{2}},
$$

where $R^{V}$ is the Chern curvature:

$$
R^{V}(X, Y) Z=D_{X}^{V} D_{Y}^{V} Z-D_{Y}^{V} D_{X}^{V} Z-D_{[X, Y]}^{V} Z .
$$

Then the Ricci curvature for $(M, F)$ is given by

$$
\operatorname{Ric}(V)=\sum_{\alpha=1}^{n-1} K\left(V, e_{\alpha}\right)
$$

where $e_{1}, \ldots, e_{n-1}, \frac{V}{F(V)}$ form an orthonormal basis of $T_{x} M$ with respect to $g_{V}$.

Given a Finsler manifold $(M, F)$, the dual Finsler metric $F^{*}$ on $M$ is defined by

$$
F^{*}\left(\xi_{x}\right)=\sup _{Y \in T_{x} M \backslash 0} \frac{\xi(Y)}{F(Y)}, \quad \forall \xi \in T^{*} M,
$$

and the corresponding fundamental tensor is defined by

$$
g^{* k l}(\xi)=\frac{1}{2} \frac{\partial F^{* 2}(\xi)}{\partial \xi_{k} \partial \xi_{l}} .
$$

The Legendre transformation $\mathcal{L}: T M \rightarrow T^{*} M$ is defined by

$$
\mathcal{L}(Y)= \begin{cases}g_{Y}(Y, \cdot), & Y \neq 0 \\ 0, & Y=0\end{cases}
$$

It is well-known that for any $x \in M$, the Legendre transformation is a smooth diffeomorphism from $T_{x} M \backslash 0$ onto $T_{x}^{*} M \backslash 0$, and it is norm-preserving, namely, $F(Y)=F^{*}(\mathcal{L}(Y)), \forall Y \in T M$. Consequently, $g_{i j}(Y)=g^{* i j}(\mathcal{L}(Y))$. 
For a smooth function $u$ on $M$, the gradient vector of $u$ at $x$ is defined by $\nabla u(x):=\mathcal{L}^{-1}(d u)$. Locally we can write in coordinates

$$
\nabla u=\sum_{i, j=1}^{n} g^{i j}(x, \nabla u) \frac{\partial u}{\partial x^{i}} \frac{\partial}{\partial x^{j}} \quad \text { in } M_{u},
$$

where $M_{u}:=\{x \in M \mid d u(x) \neq 0\}$.

A volume form $d \mu$ on $(M, F)$ is noting but a global nondegenerate $n$-form on $M$. In local coordinates we can express $d \mu$ as $d \mu=\sigma(x) d x^{1} \wedge \cdots \wedge d x^{n}$. Let $V=V^{i} \frac{\partial}{\partial x^{i}}$ be a smooth vector field on $M$. Then the divergence of $V$ with respect to $d \mu$ and the Finsler-Laplacian of $u$ are defined by

$$
\operatorname{div} V:=\sum_{i=1}^{n}\left(\frac{\partial V^{i}}{\partial x^{i}}+V^{i} \frac{\partial \log \sigma}{\partial x^{i}}\right), \quad \Delta u:=\operatorname{div}(\nabla u) .
$$

The Finsler-Laplacian is better viewed in a weak sense due to the lack of regularity, that is, for $u \in W^{1,2}(M)$,

$$
\int_{M} \phi \Delta u d \mu=-\int_{M} d \phi(\nabla u) d \mu \quad \text { for } \phi \in C_{0}^{\infty}(M) .
$$

Let $(M, F, d \mu)$ be a Finsler $n$-manifold. For $V \in T_{x} M \backslash 0$, define

$$
\tau(x, V):=\log \frac{\sqrt{\operatorname{det}\left(g_{i j}(x, V)\right)}}{\sigma(x)} .
$$

$\tau$ is called the distortion of $(M, F, d \mu)$. To measure the rate of distortion along geodesics, we define

$$
S(x, V):=\frac{d}{d t}[\tau(\dot{\gamma}(t))]_{t=0},
$$

where $\gamma:(-\varepsilon, \varepsilon) \rightarrow M$ is a geodesic with $\gamma(0)=x, \dot{\gamma}(0)=V . S$ is called the $S$-curvature (see [10]). Define

$$
\dot{S}(V):=F^{-2}(V) \frac{d}{d t}[S(\gamma(t), \dot{\gamma}(t))]_{t=0} .
$$

Then the weighted Ricci curvature of $(M, F, d \mu)$ is defined by (see [5])

$$
\left\{\begin{array}{l}
\operatorname{Ric}_{n}(V):= \begin{cases}\operatorname{Ric}(V)+\dot{S}(V), & \text { for } S(V)=0, \\
-\infty, & \text { otherwise, }\end{cases} \\
\operatorname{Ric}_{N}(V):=\operatorname{Ric}(V)+\dot{S}(V)-\frac{S(V)^{2}}{(N-n) F(V)^{2}}, \forall N \in(n, \infty), \\
\operatorname{Ric}_{\infty}(V):=\operatorname{Ric}(V)+\dot{S}(V) .
\end{array}\right.
$$

\section{Proof of Theorem 1.1}

Let $(\mathbb{R},|\cdot|, m)$ be the 1-dimensional Euclidean space with Lebesgue measure. Then $\widetilde{M}=M \times \mathbb{R}$ has the product metric $\widetilde{F}=\sqrt{F^{2}+t^{2}}$ and the volume form 
$d \tilde{\mu}=d \mu d t=\sigma(x) d x d t$. It is easy to check that $\widetilde{F}$ is a Finsler metric on $\widetilde{M}$. Moreover, we have

$$
\left(\tilde{g}_{\alpha \beta}\right)=\left(\begin{array}{cc}
g_{i j} & 0 \\
0 & 1
\end{array}\right), \quad 1 \leq \alpha, \beta \leq n+1,1 \leq i, j \leq n .
$$

Denote by $\widetilde{\nabla}, \widetilde{\Delta}$ the gradient and the Laplacian on $\widetilde{M}$, respectively. Let $f(x, t)$ be a smooth function defined on $\widetilde{M}$. Then

$$
\begin{aligned}
\widetilde{\nabla} f & =\tilde{g}^{\alpha \beta} \frac{\partial f}{\partial x^{\beta}} \frac{\partial}{\partial x^{\alpha}}=g^{i j} \frac{\partial f}{\partial x^{j}} \frac{\partial}{\partial x^{i}}+\frac{\partial f}{\partial t} \frac{\partial}{\partial t}=\nabla f+f^{\prime}(t) \frac{\partial}{\partial t} \\
\widetilde{\Delta} f & =\frac{1}{\sigma} \frac{\partial}{\partial x^{\alpha}}\left(\sigma \tilde{g}^{\alpha \beta} \frac{\partial f}{\partial x^{\beta}}\right)=\frac{1}{\sigma} \frac{\partial}{\partial x^{i}}\left(\sigma g^{i j} \frac{\partial f}{\partial x^{j}}\right)+\frac{1}{\sigma} \frac{\partial}{\partial t}\left(\sigma \frac{\partial f}{\partial t}\right) \\
& =\Delta f+f^{\prime \prime}(t) .
\end{aligned}
$$

Recall that the Christoffel symbol with respect to the Chern connection on $(\widetilde{M}, \widetilde{F})$ is (see [1])

$$
\widetilde{\Gamma}_{\beta \gamma}^{\alpha}=\frac{1}{2} \tilde{g}^{\alpha \eta}\left(\frac{\delta \tilde{g}_{\eta \beta}}{\delta x^{\gamma}}+\frac{\delta \tilde{g}_{\eta \gamma}}{\delta x^{\beta}}-\frac{\delta \tilde{g}_{\beta \gamma}}{\delta x^{\eta}}\right)
$$

where

Therefore, one obtains

$$
\frac{\delta}{\delta x^{\alpha}}=\frac{\partial}{\partial x^{\alpha}}-\widetilde{N}_{\alpha}^{\beta} \frac{\partial}{\partial y^{\beta}}, \quad \widetilde{N}_{\alpha}^{\beta}=\widetilde{\Gamma}_{\alpha \gamma}^{\beta} y^{\gamma}
$$

$$
\widetilde{\Gamma}_{\beta \gamma}^{\alpha}= \begin{cases}\Gamma_{j k}^{i}, & 1 \leq i, j, k \leq n \\ 0, & \text { otherwise }\end{cases}
$$

By a direct computation, we further have

$$
\widetilde{R}(X, Y) Z=R(X, Y) Z, \quad \widetilde{R}\left(\frac{\partial}{\partial t}, Y\right) Z=\widetilde{R}(X, Y) \frac{\partial}{\partial t}=0, \quad \forall X, Y, Z \in T M .
$$

Besides, the $S$-curvature of $\widetilde{M}$

$$
\widetilde{S}=\frac{\partial \widetilde{G}^{\alpha}}{\partial y^{\alpha}}-y^{\alpha} \frac{\partial}{\partial x^{\alpha}}(\log \sigma(x))=\frac{\partial G^{i}}{\partial y^{i}}-y^{i} \frac{\partial}{\partial x^{i}}(\log \sigma(x))=S,
$$

and $\dot{\widetilde{S}}=\dot{S}$, where $\widetilde{G}^{\alpha}=\frac{1}{2} \widetilde{\Gamma}_{\beta \gamma}^{\alpha} y^{\beta} y^{\gamma}$. Thus we still have the lower bound for the weighted Ricci curvature of $\widetilde{M}$. That is $\widetilde{\operatorname{Ric}}_{N} \geq-K$. Set

$$
f(x, t)=e^{\lambda t} u(x) .
$$

Then, from $3.2, f(x, t)$ is a positive harmonic function on $\widetilde{M}$. Namely, $\widetilde{\Delta} f=0$. By using the gradient estimate in [12], we have

$$
\begin{aligned}
\max \{F(x, \nabla & \log u(x)), F(x, \nabla(-\log u(x)))\} \\
& \leq \max \{\widetilde{F}(x, \widetilde{\nabla} \log f(x, t)), \widetilde{F}(x, \widetilde{\nabla}(-\log f(x, t)))\} \\
& \leq C\left(\frac{1+R \sqrt{K}}{R}\right) .
\end{aligned}
$$


As applications of Theorem 1.1. we give a Liouville property and a Harnack inequality in the following corollary.

Corollary 3.1. Let $(M, F, d \mu)$ be as in Theorem 1.1 with $K=0$. If $u$ is a nonnegative solution of (1.1) on $M$, then $u$ vanishes identically provided $\lambda>0$.

Proof. Assume that $u>0$. Letting $K=0$ and $R \rightarrow+\infty$ in 1.2 , we have

$$
F(x, \nabla \log u(x))=F(x, \nabla(-\log u(x)))=0,
$$

which implies that $u$ is constant. Then from equation 1.1 we get $u \equiv 0$ on $M$. This contradicts the assumption.

Corollary 3.2. Let $(M, F, d \mu)$ be as in Theorem 1.1 and $u$ be a positive solution of (1.1) in forward geodesic ball $B_{2 R}^{+}(p) \subset M$. Then there exists some constant $C=C\left(N, \Lambda_{1}, \Lambda_{2}\right)$, depending on $N$, the uniform constants $\Lambda_{1}$ and $\Lambda_{2}$, such that

$$
\sup _{B_{R}^{+}(p)} u \leq e^{C(1+\sqrt{K} R)} \inf _{B_{R}^{+}(p)} u .
$$

Proof. Choose two points $x_{1}, x_{2} \in B_{R}^{+}(p)$ such that $u\left(x_{1}\right)=\sup _{B_{R}^{+}(p)} u$ and $u\left(x_{2}\right)=$ $\inf _{B_{R}^{+}(p)} u$. Draw a minimal geodesic $\gamma$ from $x_{1}$ to $x_{2}$. Then by the triangle inequality, $\gamma \subset B_{(\eta / 2+1) R}^{+}(p)$, where $\eta$ is the reversibility of $F$. Since $F$ is uniformly smooth and uniformly convex, $\eta<+\infty$ and depends on $\Lambda_{1}$ and $\Lambda_{2}$. Therefore,

$$
\log \frac{u\left(x_{1}\right)}{u\left(x_{2}\right)}=\left|\int_{\gamma} \frac{d \log u}{d s}\right| \leq \max _{B_{(\eta / 2+1) R}^{+}} F(x, \nabla \log u(x)) \int_{\gamma} d s \leq C(1+\sqrt{K} R) .
$$

\section{Proof of Theorem 1.2}

Set

$$
G=(f+a)^{\frac{1-q}{2}}, \quad a>0, q>1 .
$$

Then $0<G \leq a^{\frac{1-q}{2}}$. Taking the differential in both sides, we have

$$
d G=-\frac{q-1}{2}(f+a)^{-\frac{q+1}{2}} d f
$$

For a positive number $\lambda$ and any smooth function $u$, we have

$$
\mathcal{L}^{-1}(\lambda d u)=\lambda \mathcal{L}^{-1}(d u)=\lambda \nabla u, \quad \mathcal{L}^{-1}(-d u)=-\overleftarrow{\nabla} u
$$

Thus, by a straight calculation we obtain

$$
\begin{aligned}
& \overleftarrow{\nabla} G=-\frac{q-1}{2}(f+a)^{-\frac{q+1}{2}} \nabla f=-\frac{q-1}{2} G^{\frac{q+1}{q-1}} \nabla f \\
& \overleftarrow{\Delta} G=-\frac{q-1}{2} G^{\frac{q+1}{q-1}} \Delta f-\frac{q+1}{2} G^{\frac{2}{q-1}} d G(\nabla f) \quad \text { on } M_{G}=M_{f}
\end{aligned}
$$

Here the notations $\overleftarrow{\nabla}, \overleftarrow{\Delta}$ denote respectively the gradient and the Finsler Laplacian with respect to the reverse Finsler metric $\overleftarrow{F}(x, y):=F(x,-y)$. It is easy to check 
that $\overleftarrow{\nabla} u=-\nabla(-u), \overleftarrow{\Delta} u=-\Delta(-u)$ for a smooth function $u$. From (4.1) and 4.2, we deduce that

$$
\frac{1-q}{2} G^{\frac{2 q}{q-1}} \Delta f=G \overleftarrow{\Delta} G-\frac{q+1}{q-1} \overleftarrow{F}(\overleftarrow{\nabla} G)^{2}
$$

which can be rewritten as

$$
\frac{\Delta f}{(f+a)^{q}}=-\frac{2}{q-1} G \overleftarrow{\Delta} G+\frac{2(q+1)}{(q-1)^{2}} \overleftarrow{F}(\overleftarrow{\nabla} G)^{2}
$$

Observe that $-G$ is bounded from above; we can then apply the Omori-Yau maximum principle $([14$, Theorem 0.3]) on $-G$. That is, there exists a point sequence $\left\{p_{k}\right\} \subset M_{G}$ such that

$$
\lim _{k \rightarrow \infty} F(\nabla(-G))\left(p_{k}\right)=0, \quad \lim _{k \rightarrow \infty} \Delta(-G)\left(p_{k}\right) \leq 0, \quad \lim _{k \rightarrow \infty}(-G)\left(p_{k}\right)=\sup _{M}(-G) .
$$

The first two formulas imply

$$
\lim _{k \rightarrow \infty} \overleftarrow{F}(\overleftarrow{\nabla} G)\left(p_{k}\right)=0, \quad \lim _{k \rightarrow \infty} \overleftarrow{\Delta} G\left(p_{k}\right) \geq 0
$$

Using 4.3,

$$
\lim _{k \rightarrow \infty} \frac{\Delta f\left(p_{k}\right)}{\left(f\left(p_{k}\right)+a\right)^{q}} \leq 0 .
$$

From the definition of $G$, we have $f\left(p_{k}\right) \rightarrow \sup _{M} f$ when $-G\left(p_{k}\right) \rightarrow \sup _{M}(-G)$. Since $\Delta f \geq c f^{d}$, we obtain

$$
\frac{c\left(\sup _{M} f\right)^{d}}{\left(\sup _{M} f+a\right)^{q}} \leq 0
$$

for $d>1$ and any $q>1$. We claim that $\sup _{M} f<+\infty$. If not, then we choose $q<d$, the left side of the above inequality is $+\infty$, which is a contradiction. Thus $\sup _{M} f<+\infty$. Using the inequality above again, we find $\sup _{M} f=0$. This means $f \equiv 0$.

If $f$ has an upper bound, the restriction on $d>1$ in 1.3 can be improved to $d>0$.

Proposition 4.1. Let $(M, F, d \mu)$ be a Finsler $n$-manifold, and $r(x)=d_{F}(p, x)$ be the distance function from a fixed point $p \in M$. Assume that the weighted Ricci curvature satisfies $\operatorname{Ric}_{N}(x, y) \geq-G^{2}(r(x)), \forall y \in T_{x} M, N \in[n, \infty)$, where $G$ is a smooth function satisfying

$$
G \geq 1, \quad G^{\prime} \geq 0, \quad \int_{0}^{\infty} \frac{d s}{G(s)}=\infty .
$$

If a nonnegative function $f \in C^{2}(M)$ bounded above satisfies

$$
\Delta f \geq c f^{d}, \quad c>0, d>0,
$$

then $f$ vanishes identically. 
Proof. Applying the Omori-Yau maximum principle ([14, Theorem 0.3]), there exists a point sequence $\left\{p_{k}\right\} \subset M_{f}$ such that

$$
\lim _{k \rightarrow \infty} f=\sup _{M} f, \quad \lim _{k \rightarrow \infty} \Delta f\left(p_{k}\right) \leq 0 .
$$

Thus,

$$
0 \geq \lim _{k \rightarrow \infty} \Delta f\left(p_{k}\right) \geq c \lim _{k \rightarrow \infty} f^{d}=c \sup _{M} f^{d} \geq 0 .
$$

Since $f \geq 0$, we have $f \equiv 0$ on $M$.

\section{References}

[1] D. Bao, S.S. Chern and Z. Shen, An introduction to Riemann-Finsler geometry, Springer, 2000. MR 1747675

[2] S.M. Choi, J.H. Kwon and Y.J. Suh, A Liouville-type theorem for complete Riemannian manifolds, Bull. Korean Math. Soc. 35 (1998), no. 2, 301-309. MR 1619292.

[3] L. Ma, Some properties of non-compact complete Riemannian manifolds, Bull. Sci. Math. 130 (2006), no. 4, 330-336. MR 2237447

[4] S. Nishikawa, On maximal spacelike hypersurfaces in a Lorentzian manifold, Nagoya Math. J. 95 (1984), 117-124. MR 0759469.

[5] S. Ohta, Finsler interpolation inequalities, Calc. Var. Partial Differential Equations 36 (2009), no. 2, 211-249. MR 2546027.

[6] S. Ohta, Uniform convexity and smoothness, and their applications in Finsler geometry, Math. Ann. 343 (2009), no. 3, 669-699. MR 2480707

[7] S. Ohta and K.T. Sturm, Heat flow on Finsler manifolds, Comm. Pure Appl. Math. 62 (2009), no. 10, 1386-1433. MR 2547978.

[8] H.B. Rademacher, A sphere theorem for non-reversible Finsler metrics, Math. Ann. 328 (2004), no. 3, 373-387. MR 2036326

[9] R. Schoen, S.T. Yau. Lectures on Differential Geometry, International Press, Cambridge, MA, 1994. MR 1333601

[10] Z.M. Shen, Volume comparison and its applications in Riemann-Finsler geometry, Adv. Math. 128 (1997), no. 2, 306-328. MR 1454401.

[11] B.Y. Wu and Y.L. Xin, Comparison theorems in Finsler geometry and their applications, Math. Ann. 337 (2007), no. 1, 177-196. MR 2262781

[12] C. Xia, Local gradient estimate for harmonic functions on Finsler manifolds, Calc. Var. Partial Differential Equations 51 (2014), no. 3-4, 849-865. MR 3268873

[13] S.T. Yau, Harmonic functions on complete Riemannian manifolds, Comm. Pure Appl. Math. 28 (1975), 201-228. MR 0431040

[14] S.T. Yin and Q. He, A generalized Omori-Yau maximum principle in Finsler geometry, Nonlinear Anal. 128 (2015), 227-247. MR 3399527.

[15] F.E. Zhang, On the maximum principle on complete Finsler manifolds, Differential Geom. Appl. 31 (2013), no. 6, 707-717. MR 3130564

[16] F.E. Zhang and Q.L. Xia, Some Liouville-type theorems for harmonic functions on Finsler manifolds, J. Math. Anal. Appl. 417 (2014), no. 2, 979-995. MR 3194525 
[17] H.C. Zhang and X.P. Zhu, Yau's gradient estimates on Alexandrov spaces, J. Differential Geom. 91 (2012), no. 3, 445-522. MR 2981845

[18] W. Zhao and Y.B. Shen, A universal volume comparison theorem for Finsler manifolds and related results, Canad. J. Math. 65 (2013), no. 6, 1401-1435 MR 3121676.

\section{S.T. Yin}

Department of Mathematics and Computer Science, Tongling University, Anhui 244000,

P.R. China

yst419@163.com

P. Zhang $\bowtie$

School of Mathematical Sciences, University of Science and Technology of China, Anhui 230026, P.R. China

panzhang@mail.ustc.edu.cn

Received: April 10, 2017

Accepted: February 2, 2018 